\title{
Processos Conscientes e Inconscientes na Produção de Falsas Memórias
}

\author{
Tin Po Huang ${ }^{1}$ \\ Gerson Américo Janczura \\ Universidade de Brasília
}

\begin{abstract}
RESUMO - Este estudo verificou o efeito do tipo de teste e tempo de apresentação dos estímulos durante a codificação na produção de falsas memórias utilizando o paradigma DRM. Quatro intervalos de apresentação foram manipulados: 20ms, 250ms, $1000 \mathrm{~ms}$ e 3000ms. Cem estudantes universitários participaram de cada teste. Foram testadas duas hipóteses: intervalos de tempo maiores produziriam maiores índices de falsas memórias, independentemente do teste, e o teste direto produziria mais falsas memórias. Os resultados confirmaram as hipóteses e mostraram que os fatores produziram efeitos significativos nas taxas de falsas memórias. Isso sugere que, embora processos inconscientes possam estar envolvidos no fenômeno, há uma participação predominante de processos conscientes na produção dessas ilusões de memória e que esse fenômeno pode ser explicado por modelos duais de processamento que consideram tanto a codificação quanto a evocação nas falsas memórias.
\end{abstract}

Palavras-chave: falsas memórias; reconhecimento; identificação perceptual; processos conscientes; processos inconscientes.

\section{Conscious and Unconscious Processes in the Production of False Memories}

\begin{abstract}
This study investigated the effects of stimulus presentation time and type of test (recognition and perceptual identification) on false memories in the DRM paradigm. Four presentation times were manipulated during the encoding phase: $20 \mathrm{~ms}$, $250 \mathrm{~ms}, 1000 \mathrm{~ms}$ and $3000 \mathrm{~ms}$. A hundred undergraduate students participated in each test. The following hypotheses were tested: longer presentation durations should produce higher levels of false memories, regardless of memory test, and significant higher level of false memories should be observed in the direct test. Both variables produced significant effects on false memories. The results suggested that conscious processes played a major role in the production of memory illusions, with mild participation of unconscious processes. The phenomenon is discussed adopting a dual processing model that includes both encoding and retrieval processes.
\end{abstract}

Key words: false memories; recognition; perceptual identification; conscious processes; unconscious processes.

Nossas recordações podem nos iludir? Apesar de estarmos, em geral, relativamente seguros da acurácia de nossas memórias, pesquisas recentes na área de falsas memórias indicam que, sob algumas condições, as "certezas" de recuperação de eventos podem ser enganadoras (Roediger \& McDermott, 1995). Há indicações de que processos de aprendizagem poderão ocorrer em condições em que as palavras são quase imperceptíveis ou "invisíveis" ao olho humano (Seamon, Luo \& Gallo, 1998). As falsas memórias se referem às lembranças de eventos que na realidade não ocorreram ou a recordação de eventos de uma forma bastante diferente de como realmente aconteceram (Roediger \& McDermott, 1995). Roediger (1996) refere-se ao fenômeno como "ilusões de memória" ao traçar um paralelo com as mais conhecidas ilusões da percepção. Segundo o autor, as ilusões de memória correspondem a situações em que a recordação de uma lembrança passada é seriamente distorcida do evento original, que realmente aconteceu.

Em 1995, Roediger e McDermott propuseram um método, criado inicialmente por Deese, em 1959, que induz as pessoas a se convencerem de que tiveram uma experiência como, por exemplo, ver uma palavra que na verdade nunca ocorreu. Esse método ficou conhecido como o paradigma

1 Endereço: Universidade de Brasília, Instituto de Psicologia, Laboratório de Processos Cognitivos/PPB, Campus Universitário Darcy Ribeiro, Brasília, DF, Brasil 70910-900.E-mail: tinhuang9@gmail.com
DRM. No paradigma, o experimentador apresenta uma lista de palavras semanticamente relacionadas como costura, linha, ponta, furar, espetar, injeção, bordar e palheiro. Depois, em um teste de memória, os participantes devem reconhecer quais palavras foram apresentadas anteriormente. Nessa etapa, incluem-se palavras apresentadas na etapa de estudo (por exemplo, costurar e bordar) e palavras novas (por exemplo, agulha, porta e doce). A palavra agulha é denominada de palavra crítica não apresentada, porque traduz a essência semântica da lista que foi experienciada e é a palavra que está mais fortemente associada à lista. Na maioria das vezes, as pessoas lembram corretamente de ter visto costura e bordar e não ter visto porta e doce. Mais interessante, ainda, é o fato de os indivíduos freqüentemente estarem convictos de que leram também a palavra agulha, que não foi apresentada na etapa de estudo. Esse método tem se mostrado muito eficaz na indução, especialmente em nível experimental, de falsas lembranças (Payne, Elie, Blackwell \& Neuschatz, 1996; Roediger \& McDermott, 1995; Stadler, Roediger \& McDermott, 1999; Zeelenberg, Plomp \& Raaijmakers, 2003).

$\mathrm{O}$ teste de reconhecimento tem sido freqüentemente associado ao paradigma DRM, pois permite a exploração da técnica "lembrar/saber" (remember e know) proposta por Tulving (1985). Essa técnica contribui para a compreensão da questão sobre a ativação consciente ou não, durante a fase de codificação, da informação estudada. A experiência de lembrar é definida como "reviver" mentalmente a experiência, ou 
seja, reviver se refere ao fato de o participante lembrar de um aspecto físico, algum estímulo associado com a experiência ou o que estava fazendo quando ouviu ou leu a palavra-alvo. Um julgamento de saber deve ser produzido quando o indivíduo está confiante que o item ocorreu, mas não consegue reexperienciar (lembrar) a sua ocorrência. Assim, o ato de lembrar indicaria uma revivência mental da experiência e o ato de saber não (Rajaram, 1993; Roediger \& McDermott, 1995). A principal razão para o uso desse procedimento é verificar se os participantes que falsamente reconheceram as palavras críticas não apresentadas apresentariam um julgamento de lembrar, evidenciando que eles "reviveram" experiências que nunca ocorreram. Vários experimentos sobre falsos reconhecimentos, utilizando o julgamento lembrar-saber, verificaram que os falsos reconhecimentos estiveram associados com julgamentos de saber (Gardiner, 1988; Jones \& Roediger, 1995). Outros indicaram que houve uma tendência de lembrar as palavras críticas (Roediger \& McDermott, 1995; Seamon \& cols., 1998).

\section{Falsas memórias em medidas indiretas e priming}

Nos estudos utilizando o paradigma DRM, com testes diretos de recuperação livre e reconhecimento, verifica-se que os participantes tratam a palavra crítica não apresentada como se fosse uma palavra estudada (Roediger \& McDermott, 1995; Stadler \& cols., 1999). Entretanto, poucas pesquisas têm evidenciado esse efeito, utilizando testes indiretos. A investigação tradicional sobre falsas memórias tem enfatizado a recuperação livre e o reconhecimento, em que os participantes são solicitados a reportar conscientemente algum episódio ou informação específica. Mas a memória humana também se manifesta de uma forma não deliberada ou "implícita" (Graf \& Schacter, 1985). Já se têm evidências de que a palavra crítica se "comporta" como uma palavra estudada em testes de memória explícita, mas não há evidências suficientes de que essa afirmação pode ser estendida aos testes de memórias implícitas. É razoável esperar que, num teste perceptual de memória implícita, a palavra crítica apresentasse níveis similares de priming às palavras estudadas? Para responder a essa pergunta, é necessário investigar uma questão ainda não resolvida na literatura: se a palavra crítica não apresentada é ativada conscientemente ou não durante a fase de estudo. Um dos primeiros pesquisadores que tentou responder a essa indagação foi Underwood (1965). Nesse estudo, aplicou-se um teste de reconhecimento em que os participantes deveriam decidir se as palavras apresentadas no teste tinham sido estudadas anteriormente. Quando uma das palavras testadas tinha uma relação associativa com uma palavra estudada (e.g., o participante tinha que responder se a palavra cadeira tinha sido estudada quando, na verdade, a palavra mesa fora estudada), houve maior probabilidade de os indivíduos apresentarem falsas memórias em comparação a palavras testadas que não tinham qualquer associação com a palavra estudada. Underwood (1965) interpretou esse fenômeno de falso reconhecimento propondo a hipótese da ativação implícita. Essa hipótese afirma que, durante a fase de estudo, os participantes poderiam ativar conscientemente as palavras críticas e depois, na fase de teste, confundi-las com as palavras realmente estudadas. As palavras críticas poderiam ser ativadas inconscientemente numa exposição de lista de palavras semanticamente relacionadas. Nessa condição de múltipla exposição, a palavra crítica seria "reativada" automaticamente várias vezes, embora não ultrapassando o limiar para ser reconhecida conscientemente. Esse processo de reativação da palavra crítica, na fase de estudo, poderia sugerir que processos não conscientes seriam responsáveis pelas ilusões de memória. Por exemplo, quando o participante estudava as palavras mesa, móvel e banco, a palavra cadeira seria ativada e falsamente reconhecida na fase de teste. Estudos posteriores verificaram que, aumentando-se o número de palavras relacionadas semanticamente na fase de estudo, maiores são as chances de o indivíduo produzir as palavras críticas não estudadas (Hall \& Kozloff, 1973; Hintzman, 1988; Shiffrin, Huber \& Maritelli, 1995).

\section{Efeito do tempo de apresentação na produção de falsas memórias}

A manipulação do tempo de exposição dos estímulos durante a etapa de estudo é útil para ilustrar como a ativação e o monitoramento podem influenciar as falsas memórias no paradigma DRM. No experimento de McDermott e Watson (2001), foi utilizado o teste direto de recuperação livre e manipulou-se o tempo de estudo em cinco intervalos $(20 \mathrm{~ms}$, $250 \mathrm{~ms}, 1000 \mathrm{~ms}, 3000 \mathrm{~ms}$ e $5000 \mathrm{~ms}$ ). Os resultados evidenciaram que, nos tempos rápidos (20ms e $250 \mathrm{~ms}$ ), houve um aumento da média de falsas memórias e, nos tempos mais longos (1000ms, 3000ms e 5000ms), houve uma diminuição de palavras falsamente reconhecidas. Segundo McDermott e Watson, o modelo hipotético duplo de ativação e monitoramento propõe que, nos tempos rápidos de apresentação, a ativação aumentaria com o aumento de tempo, produzindo, assim, um aumento também da falsa recuperação em função da falta de controle consciente. Por outro lado, nos tempos mais longos, os participantes teriam utilizado estratégias mentais conscientes que interferiram nos efeitos da ativação espalhada. Esse resultado que delineia uma curva na forma de "U" invertido corroboraria, assim, a necessidade de se utilizar um modelo dual explicativo para o fenômeno das falsas memórias.

Três predições lógicas podem ser previstas quando se consideram testes perceptuais implícitos: primeiro, se as palavras críticas não apresentadas são tratadas como se tivessem sido apresentadas, pode-se predizer que ocorrerá priming com magnitude semelhante às palavras estudadas (McDermott, 1996; Roediger \& McDermott, 1995). Segundo, se as palavras críticas forem ativadas conscientemente durante a fase de estudo, então deveria ser observado um modesto efeito de priming, ou seja, para ocorrer uma pré-ativação da palavra, é necessário que o indivíduo ative conscientemente a palavra durante a fase de codificação. E, terceiro, se não houver nenhuma ativação consciente dessas palavras na fase de estudo, não ocorrerá priming perceptual das palavras $(\mathrm{McDermott}$, 1997). Ressalta-se que essas predições foram testadas em estudos em que o tempo de apresentação era fixo, acima de $1500 \mathrm{~ms}$. Em um estudo pioneiro realizado para tentar responder a essas hipóteses, McDermott (1997) investigou, utilizando o paradigma DRM, se ocorreria priming em dois tipos de testes indiretos e obteve como resultado priming das 
palavras críticas não apresentadas na completação de fragmentos de palavras e na completação de radicais. Os resultados não foram conclusivos quanto à magnitude do priming, ou seja, o priming das palavras críticas não foi equivalente ao das palavras estudadas. Porém, os resultados foram consistentes com a predição de que a palavra crítica não apresentada é ativada conscientemente durante a fase de estudo.

Somente a ativação implícita semântica não produz, geralmente, priming perceptual, porque não se espera priming perceptual se as palavras forem ativadas inconscientemente. Esse resultado é consistente com outras pesquisas que têm relatado um alto nível de julgamentos de lembrar das palavras críticas não apresentadas em que os participantes afirmaram, com convicção, que lembraram de terem lido as palavras críticas na fase de estudo (Roediger \& McDermott, 1995; Schacter, Verfaellie \& Pradere, 1996). Mas essas três predições descritas anteriormente não foram manipuladas com o tempo de apresentação. Se fosse feito com essa variável, esses resultados se replicariam como nos estudos anteriores?

O objetivo do presente trabalho foi verificar a ocorrência de falsas memórias em dois tipos de testes: reconhecimento e identificação perceptual, manipulando-se quatro diferentes intervalos de tempo de estudo. A manipulação do tempo de apresentação poderá contribuir para a questão sobre a evocação deliberada e a evocação implícita nas falsas memórias. Se forem observadas produções semelhantes de falsas memórias tanto em tempos rápidos quanto em tempos lentos, então será razoável afirmar que falsas memórias ocorrem tanto com a participação de processos conscientes quanto inconscientes da memória. Por outro lado, se for observado que a ocorrência de falsas memórias varia em função do tempo de apresentação da informação, ou seja, a sua probabilidade de ocorrência é maior na medida em que se aumenta o tempo de apresentação, será razoável sustentar a hipótese de que o processamento consciente desempenha um papel dominante na produção dessas ilusões de memória. Este estudo investigou as seguintes hipóteses gerais: primeiro, será evidenciado um aumento das falsas memórias com o aumento de intervalo de tempo, sugerindo, assim, que a produção de falsas memórias depende de um fator predominantemente consciente para a sua ocorrência, independentemente do tipo de teste. Segundo, haverá maior produção de falsificação de memória no teste direto de reconhecimento, porque esse teste envolve evocação consciente. Acrescentando a isso, foram testadas duas hipóteses secundárias: a) no tempo de apresentação muito breve (20ms), o qual reduz a possibilidade de envolvimento de processos conscientes na codificação, não haveria diferença significativa na produção de falsas memórias em função do tipo de teste de memória. Nesse caso, espera-se uma produção baixa de falsas memórias em ambos os testes. Isso pode ser explicado à luz da teoria da Ativação e Monitoramento (McDermott \& Watson, 2001), uma vez que tempos rápidos diminuem a probabilidade de uma ativação da rede semântica, ou seja, a palavra crítica não consegue, na maioria das vezes, ser ativada a ponto de ser confundida na fase de teste com itens estudados; e b) nos tempos mais longos, de 1000ms e $3000 \mathrm{~ms}$, que permitem maior engajamento de processamento consciente da informação, prevê-se que a produção de falsas memórias será menor no intervalo de tempo de $3000 \mathrm{~ms}$, no teste de reconhecimento do que no intervalo de $1000 \mathrm{~ms}$.
Pode-se supor, então, que, nessas condições (de tempo mais longo), a produção de falsas memórias depende de uma evocação consciente.

\section{Experimento 1}

Esse experimento teve dois objetivos: primeiro, investigar se a manipulação de quatro tempos de apresentação dos estímulos na fase de estudo $(20 \mathrm{~ms}, 250 \mathrm{~ms}, 1000 \mathrm{~ms}$ ou $3000 \mathrm{~ms}$ ), no teste de reconhecimento, influenciaria a magnitude das falsas memórias no paradigma DRM. Segundo, replicar e estender os resultados da literatura relacionados à duração do tempo de apresentação dos estímulos (Arndt \& Hirshman, 1998; Seamon \& cols., 1998). Com o objetivo de contribuir para um melhor entendimento dos mecanismos que são responsáveis pelo falso reconhecimento no respectivo paradigma, verifica-se a necessidade de um número maior de resultados que manipulem o tempo de apresentação em testes de reconhecimento. Assim, esse experimento testou se haveria um aumento na produção de falsos reconhecimentos em função do aumento do intervalo de tempo de estudo de palavras. Evidências favoráveis a essa hipótese irão sugerir que aumentos no tempo de estudo permitiriam maior ocorrência de processos de monitoramento estratégicos que são responsáveis pela diminuição da produção de falsos reconhecimentos atribuídos, normalmente, a uma ativação espalhada automatizada (McDermott \& Watson, 2001). Avaliou-se, também, se as palavras críticas apresentariam um padrão de desempenho semelhante às palavras estudadas, corroborando, assim, resultados já obtidos na literatura (Roediger \& McDermott, 1995). Por fim, esperou-se observar um aumento dos julgamentos de lembrar para as palavras críticas com o aumento de tempo do estudo, pois se supõe uma maior participação do processamento consciente que é responsável pelas escolhas em julgamentos de lembrar, em intervalos de tempos maiores.

\section{Método}

\section{Participantes}

Participaram, voluntariamente, 100 alunos graduandos de três instituições de Ensino Superior de Brasília, sendo 25 homens e 75 mulheres na faixa etária entre 17 e 60 anos $(D P=10,70$ anos $)$.

\section{Delineamento}

Aplicou-se um delineamento entre-sujeitos, no qual o fator tempo de apresentação do estímulo foi manipulado em quatro níveis: $20 \mathrm{~ms}, 250 \mathrm{~ms}, 1000 \mathrm{~ms}$ e $3000 \mathrm{~ms}$. Os participantes foram designados aleatoriamente para cada condição experimental. A variável dependente foi o desempenho no teste de reconhecimento, no qual se avaliou a ocorrência de falsas memórias, erros e acertos (memórias verdadeiras).

\section{Materiais}

Foram utilizadas 10 listas de palavras semanticamente relacionadas, das normas de Stein e Pergher (2001), que é 
uma versão traduzida e adaptada para o português das normas em inglês de Stadler e cols. (1999). Cada lista continha 15 palavras associadas, perfazendo um total de 150 palavras. Além dessas palavras, cada lista incluía uma palavra crítica, que traduzia a essência semântica da lista, sendo aquela que estava mais fortemente associada à lista. Um exemplo de lista usada foi sorriso, felicidade, festa, vida, amigos, família, paz, nascimento, filhos, euforia, pular, diversão, sucesso, carnaval e gol, sendo a palavra crítica não apresentada alegria. As listas usadas são identificadas pelas seguintes palavras críticas: alegria, bandeira, carro, exército, fumar, aranha, montanha, pé, mágoa e rei.

Além das listas acima, foram utilizadas, na fase de treino, seis palavras semanticamente relacionadas: cadeira, sentar, mesa, madeira, objeto e sala. Essas palavras não tinham relação semântica com aquelas da fase de estudo.

$\mathrm{Na}$ fase de teste, foram apresentadas 60 palavras, das quais 30 eram das listas de palavras estudadas (três palavras de cada uma das 10 listas estudadas) e 30 correspondiam a palavras novas, não estudadas. As três palavras de cada lista foram selecionadas em função da posição que ocupava na lista original, sendo, respectivamente, as posições seriais $1^{a}$, 8 e 10 10 . A razão dessas posições foi incluir palavras que exibiam alta, média e baixa associação semântica com a palavra crítica. Destas, 20 foram selecionadas de 10 outras listas de Stein e Pergher (2001) e 10 eram as palavras críticas, não apresentadas, das listas estudadas.

\section{Procedimentos}

As sessões experimentais foram conduzidas em ambientes isolados nas instituições de Ensino Superior. Utilizou-se um computador para a apresentação dos estímulos das fases de estudo e teste. As sessões experimentais duraram, em média, 12 minutos. As instruções explicavam aos participantes que se tratava de um estudo sobre memória e a tarefa era memorizar palavras que seriam apresentadas na tela do computador, uma de cada vez, rapidamente. Após essas instruções, os participantes recebiam um consentimento informado ${ }^{2}$ para assinar caso aceitassem continuar o estudo. O tempo de apresentação de cada palavra foi equivalente ao tempo da condição em que cada participante fora designado $(20 \mathrm{~ms}, 250 \mathrm{~ms}, 1000 \mathrm{~ms}$ ou $3000 \mathrm{~ms}$ ). Todas as palavras foram apresentadas na fonte System, tamanho 16. Após a leitura das instruções, o experimentador iniciava a apresentação das 10 listas de palavras em uma ordem randômica. As 15 palavras de cada lista foram apresentadas uma de cada vez, numa ordem fixa e decrescente de associação semântica com a palavra crítica. Segundo as normas de Stein e Pergher (2001), a posição serial da palavra na lista corresponde à magnitude da força associativa semântica, sendo que palavras mais fortemente associadas ocupam os primeiros lugares de cada lista e as mais fracamente os últimos lugares. O tempo do intervalo entre estímulos era de $32 \mathrm{~ms}$ nas quatro condições experimentais. Após a apresentação das listas, uma atividade distratora era administrada, que consistia em executar, durante dois minutos, operações aritméticas de multiplicação, adição e subtração. A função da atividade distratora era controlar os efeitos de recência

2 Segundo as diretrizes e normas éticas da Resolução 196/1996 envolvendo pesquisas com Seres Humanos. e primazia da ordem de apresentação dos estímulos do estudo. Após essa etapa, os participantes foram submetidos ao teste de reconhecimento no qual as palavras foram apresentadas na tela, uma de cada vez, e os participantes deveriam ler em voz alta e identificar se as mesmas haviam sido apresentadas na fase de estudo. Os participantes deveriam responder "sim" se julgava ter visto a palavra ou responder "não" se julgavam não tê-la lido. Se respondessem "sim", teriam que fazer também um julgamento de "lembrar/saber", ou seja, responder "sim", eu "lembro", ou "sim", eu "sei”. O tempo para o julgamento após a apresentação de cada palavra era livre. As respostas dos participantes foram registradas numa folha pelo experimentador.

\section{Resultados}

As palavras utilizadas neste estudo foram separadas em três categorias: palavras críticas não apresentadas (PC), palavras apresentadas na fase de estudo (PE) e palavras novas não apresentadas (PN), que não tinham relação semântica com as palavras estudadas. Os efeitos do tempo de apresentação e da categoria de palavras no teste de reconhecimento foram avaliados por uma análise da variância mista para dois fatores. A ANOVA revelou um resultado significativo para o tempo de apresentação, $F(3,96)=67,142, M S=17702,753, p=0,000$, para a categoria de palavra, $F(3,96)=17,427, M S=6976,593$, $p=0,000$, e também para a interação entre esses fatores, $F(6$, 192)=4,838, $M S=1936,642, p=0,001$. As médias percentuais do reconhecimento de PE em função do tempo de apresentação das palavras que foram estudadas foram 47,2\%, 67,2\%, $78,5 \%$ e $80,8 \%$ para os tempos de $20 \mathrm{~ms}, 250 \mathrm{~ms}, 1000 \mathrm{~ms}$ e $3000 \mathrm{~ms}$, respectivamente (ver os dados da Tabela 1 sobre Reconhecimento). O teste subseqüente feito para comparação entre médias (Tukey, $p<0,05$ ) indicou que todas as comparações entre médias foram significativas, excetuando a comparação entre $1000 \mathrm{~ms}$ e $3000 \mathrm{~ms}$. A Tabela 1 , que apresenta as médias dos dois experimentos e é disponibilizada no próximo experimento, revela que a maior diferença na porcentagem de acertos das PC ocorreu entre $20 \mathrm{~ms}$ e $3000 \mathrm{~ms}(58,8 \%)$ e que o índice de acertos aumentou em função do aumento do tempo de apresentação das palavras. Esse resultado corrobora a hipótese prevista para o efeito principal desse fator.

Uma ANOVA mista para dois fatores analisou a influência do tempo de apresentação e da categoria de palavras nos julgamentos de lembrar. O tempo de apresentação $[F(3$, 96)=19,305, $M S=12330,960, p=0,000]$ e a categoria de palavras $[F(3,96)=272,268, M S=42990,583, p=0,000]$ produziram efeitos significativos nos julgamentos de lembrar. Também foi significativa a interação entre os fatores, $F(6$, 192) $=34562, M S=5456,810, p=0,000$. As médias percentuais de respostas de lembrar em função do tempo de apresentação das palavras foram $15,4 \%, 33,6 \%, 42,6 \%$ e $42,5 \%$ para os tempos de $20 \mathrm{~ms}, 250 \mathrm{~ms}, 1000 \mathrm{~ms}$ e $3000 \mathrm{~ms}$, respectivamente. A Figura 1 evidencia a interação entre o tempo de apresentação e a categoria de palavra.

A Figura 1 mostra uma diferença acentuada (32\%) na média de respostas de "lembrar", entre o tempo mais breve e o tempo mais longo, e os julgamentos de lembrar apresentam um desempenho semelhante aos da categoria de PC (produção de falsas memórias), enfim, os julgamentos de lembrar aumentam com 
o aumento do tempo de apresentação. Nas categorias de PC e $\mathrm{PE}$, verifica-se um desempenho semelhante nos julgamentos de lembrar. Comparações subsequientes entre as médias da interação desses tipos de palavras versus tempo de apresentação confirmaram a significância entre todas as diferenças, excetuando os tempos $1000 \mathrm{~ms}$ e $3000 \mathrm{~ms}$ ( $p=0,987)$, para essas duas categorias.

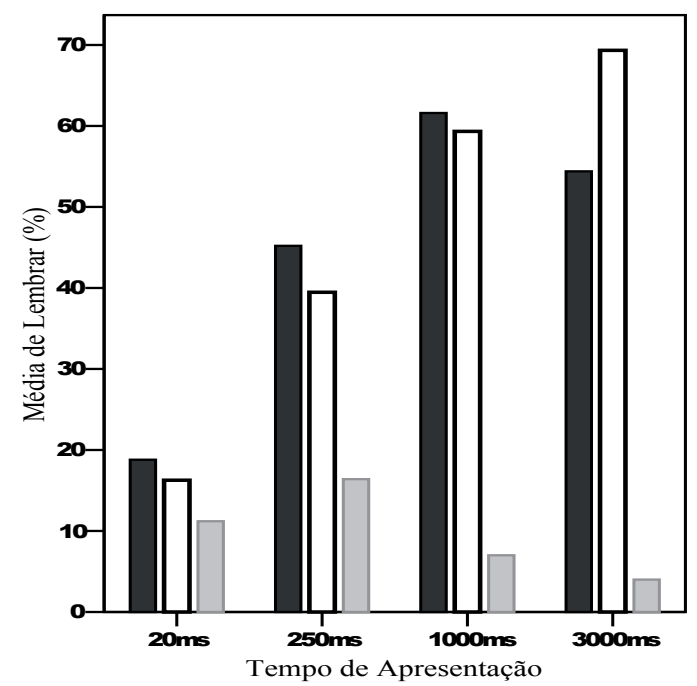

$\square$ PC
$\square$ PE
$\square$ PN

Figura 1. Médias das porcentagens do julgamento de "lembrar" em função do tempo de apresentação e categoria de palavras.

\section{Experimento 2}

O segundo experimento teve como principal objetivo avaliar a ocorrência de priming das palavras críticas não apresentadas, ou seja, a produção de priming de falsas memórias em um teste perceptual implícito (identificação perceptual) em função de quatro tempos de apresentação dos estímulos.

A hipótese testada neste experimento é de que a magnitude de priming das falsas memórias aumentaria com o aumento do tempo de estudo (de $20 \mathrm{~ms}$ a $3000 \mathrm{~ms}$ ) e não seriam observadas diferenças significativas entre as exposições mais longas (1000ms e $3000 \mathrm{~ms}$ ), sendo que, no intervalo de $20 \mathrm{~ms}$, o priming deverá ser modesto ou ausente. A explicação para essa expectativa é que, no intervalo mais breve, o indivíduo não dispõe de tempo suficiente para processar conscientemente as palavras na fase de estudo, utilizando, por exemplo, os processos de monitoramento ou alguma outra estratégia mental.

A hipótese desse experimento está relacionada às previsões descritas anteriormente, ou seja, será observada uma média semelhante de priming entre as palavras críticas e as palavras estudadas e, se as palavras críticas forem ativadas conscientemente na fase de estudo, então deverá haver uma modesta quantidade de priming das mesmas. A confirmação dessa hipótese corroborará o argumento de que o fenômeno das falsas memórias pode ser promovido pelo priming, ou seja, sem a participação obrigatória de processos conscientes na fase de teste.

\section{Método}

\section{Participantes e delineamento}

Participaram, voluntariamente, 100 alunos universitários de quatro instituições de Ensino Superior de Brasília, sendo
28 homens e 72 mulheres na faixa etária entre 18 e 53 anos $(D P=9,04)$. O delineamento administrado neste experimento foi idêntico ao anterior.

\section{Materiais}

Este experimento utilizou as mesmas listas do experimento anterior tanto na fase de estudo (150 palavras distribuídas em 10 listas) quanto na do teste (60 palavras). Para a fase de calibração, foram acrescentadas 70 palavras. Essas palavras eram formadas de seis letras e foram selecionadas, aleatoriamente, das normas de freqüência de ocorrência de palavras de Pinheiro (1996). A média de freqüência de ocorrência das palavras era $68,98(D P=27,69)$ por 10.000 palavras.

\section{Procedimentos}

A estrutura das sessões experimentais foi semelhante ao procedimento do Experimento 1, excetuando-se, apenas, a inserção de uma fase de calibração antes da fase de estudo. O objetivo dessa etapa era familiarizar o participante com o teste de identificação perceptual e estabelecer uma velocidade de projeção das palavras para o teste. $\mathrm{O}$ critério usado para determinar a duração do tempo de projeção requerido para cada participante foi aquele no qual o indivíduo atingisse, aproximadamente, $50 \%$ de acertos na identificação perceptual das palavras (Hunt \& Toth, 1990; Jacoby, 1983; Jacoby \& Hollingshead, 1989; Nairne, 1988; Ratcliff, McKonn \& Verwoerd, 1989). Utilizou-se uma máscara, com duração de 700ms, constituída de barras paralelas que teve a finalidade de impedir o processamento visual da palavra projetada além do intervalo de tempo preestabelecido. O término da apresentação da máscara era seguido de um sinal de interrogação ("?") no centro da tela. Esse sinal indicava que o participante deveria verbalizar a palavra lida. No caso de dúvidas, os participantes foram instruídos a "chutar" a palavra lida, ou seja, responder a primeira palavra que viesse à mente, justamente para verificar efeitos possíveis de priming. As 10 primeiras palavras apresentadas tinham as respectivas durações de projeções: $100 \mathrm{~ms}, 100 \mathrm{~ms}, 100 \mathrm{~ms}, 100 \mathrm{~ms}, 90 \mathrm{~ms}$, $80 \mathrm{~ms}$, 70ms, $60 \mathrm{~ms}$, $50 \mathrm{~ms}$ e $45 \mathrm{~ms}$. A diminuição gradual do tempo de projeção tinha o objetivo de familiarizar o individuo com a rapidez da apresentação e detectar o menor liminar de percepção de leitura das palavras. Foram estabelecidos três critérios hierárquicos para se estabelecer a velocidade de projeção para cada indivíduo: primeiro, a velocidade de projeção de cada participante corresponderia àquela na qual o mesmo conseguia identificar corretamente $50 \%$ das palavras. No caso de observar-se esse índice em mais de um intervalo de tempo, optou-se por escolher o menor intervalo de tempo. Segundo, nos casos em que os participantes não alcançassem os $50 \%$, seria estabelecido valor intermediário entre dois tempos como, por exemplo, se o participante tivesse $60 \%$ de acertos no tempo de $40 \mathrm{~ms}$ e $40 \%$ de acertos no tempo de $35 \mathrm{~ms}$, se optaria por $37 \mathrm{~ms}$. E terceiro, se o participante tivesse menos do que $30 \%$ de acertos na duração mais longa (40ms), seria utilizado o tempo de $45 \mathrm{~ms}$ no teste de identificação perceptual. Por outro lado, se o mínimo de acertos fosse maior que $70 \%$ na duração $20 \mathrm{~ms}$, seria aplicado um outro bloco com a duração de $15 \mathrm{~ms}$ por palavra, para 
atingir-se o critério de acertos próximo de 50\%, seguindo, novamente, os critérios descritos acima. Na fase de teste, os participantes foram submetidos a um teste de identificação perceptual, no qual apresentaram-se 60 palavras em uma ordem randômica. Desse total, 30 palavras correspondiam a palavras já lidas na fase de estudo e 30 a palavras novas, das quais 10 correspondiam às palavras críticas e 20 às palavras distratoras. O procedimento de apresentação de cada palavra foi semelhante ao da fase de calibração, na qual o participante tinha que ler cada palavra que foi projetada, com a diferença de que o tempo de projeção da palavra era fixa, ou seja, aquele determinado na fase de calibração para cada participante. A distância fixada entre os indivíduos e a tela de computador foi de $55 \mathrm{~cm}$. Essa distância foi mantida por um anteparo no qual o participante posicionava a sua cabeça na frente da tela do computador.

\section{Resultados}

Uma análise da variância mista para dois fatores verificou o efeito do tempo de apresentação e da categoria de palavras no teste de identificação perceptual. $\mathrm{O}$ tempo de apresentação $[F(3,96)=11,040, M S=2160,182, p=0,0000]$ e a categoria de palavras $[F(3,96)=10,059 M S=1206,954 p=0,0001]$ produziram efeitos significativos na identificação perceptual. A interação entre os fatores não foi significativa.

As médias percentuais de corretas identificações em função do tempo de apresentação dos estímulos na fase de estudo foram $41 \%, 45,7 \%, 51,7 \%$ e $52,1 \%$ para os intervalos de tempo $20 \mathrm{~ms}, 250 \mathrm{~ms}, 1000 \mathrm{~ms}$ e $3000 \mathrm{~ms}$, respectivamente. O teste subseqüente para comparação entre médias (Tukey, $p<0,05)$ indicou que todas as comparações foram significativas, excetuando a comparação entre os intervalos de tempo $1000 \mathrm{~ms}$ e $3000 \mathrm{~ms}$. Destaca-se a diferença de acertos entre os intervalos de tempo $20 \mathrm{~ms}$ e $3000 \mathrm{~ms}$, indicando que a manipulação do tempo de apresentação afetou a produção, mas de uma forma não tão expressiva quanto no teste direto.

Um dos objetivos deste estudo foi comparar a produção de falsas memórias em função de diferentes testes de memória. A fim de analisar a influência desse fator, foi calculada uma ANOVA mista para três fatores, tratando como variáveis entre-sujeitos, o tipo de teste e o tempo de apresentação das palavras e como fator intra-sujeitos, a categoria de palavra. O fator tipo de teste $[F(1,192)=280,199, M S=64397,76$, $p=0,000$ ] produziu efeito significativo no desempenho, isto é, foi mais provável que os participantes recordassem no teste de reconhecimento (média=68,4\%) do que na identificação perceptual (média $=47,7 \%$ ). Também foram significativas as interações entre tipo de teste e tempo de apresentação $[F(3$, 192) $=16,880, M S=3879,58, p=0,000]$, entre tipo de teste e categoria de palavra $[F(2,384)=24,265, M S=6309,18, p=0,000]$ e entre os três fatores, $F(6,384)=3639, M S=946,145$, $p=0,0016$. Em relação a essa interação, a análise dos contrastes revelou que: a) as palavras críticas foram lembradas com maior probabilidade no reconhecimento (média $=63,7 \%$ ) do que na identificação perceptual (média $=51,5 \%$ ), $[F=29,774$, $p=0,000]$; b) as palavras estudadas também tiveram maior probabilidade de recordação no teste direto (média=63,6\%) do que no indireto (média=47,1\%), [F=54,527, $p=0,000]$, e, similarmente, c), palavras não estudadas tiveram desempenho melhor no reconhecimento (média $=78,1 \%$ ) do que na identificação perceptual (média=44,7\%), $[F=223,824, p=0,000]$. A Tabela 1 apresenta os resultados em função do teste de reconhecimento (Experimento 1) e Identificação Perceptual (Experimento 2), do tipo de palavra (PC, PE, PN) e do tempo de apresentação.

Tabela 1. Média de Acertos (\%) das categorias de palavras em função do tipo de teste e do tempo de apresentação.

\begin{tabular}{lllll}
\hline Tipo de Teste & \multirow{2}{*}{ Tempo de apresentação } & \multicolumn{3}{c}{ Categoria de palavra } \\
& & PC & PE & PN \\
\hline Reconhecimento & $20 \mathrm{~ms}$ & 38,0 & 33,6 & 70,0 \\
(Experimento 1) & $250 \mathrm{~ms}$ & 68,0 & 65,3 & 68,4 \\
& $1000 \mathrm{~ms}$ & 77,6 & 74,9 & 83,2 \\
& $3000 \mathrm{~ms}$ & 71,2 & 80,4 & 90,8 \\
& Média & 63,7 & 63,5 & 78,1 \\
Identificação & $20 \mathrm{~ms}$ & 44,8 & 40,1 & 38,2 \\
Perceptual & $250 \mathrm{~ms}$ & 49,6 & 45,0 & 42,6 \\
(Experimento 2) & $1000 \mathrm{~ms}$ & 55,6 & 51,4 & 49,0 \\
& $3000 \mathrm{~ms}$ & 56,0 & 51,6 & 48,8 \\
& Média & 51,5 & 47,0 & 44,6 \\
\hline
\end{tabular}

Legenda: $\mathrm{PC}=$ palavras críticas, $\mathrm{PE}=$ palavras estudadas, $\mathrm{PN}=$ palavras novas, não estudadas.

A Tabela 1 mostra que os acertos de PC são muito semelhantes aos de PE nos dois tipos de testes e não há diferença significativa entre essas palavras em função do mesmo intervalo de tempo. Esse fato indica a produção de falsas memórias mesmo nos tempos mais longos. Por fim, a influência do tempo de apresentação é mais homogênea na identificação perceptual em função do tipo de palavra, quando se consideram aumentos gradativos no tempo de estudo de palavras.

\section{Discussão Geral}

Este estudo confirmou a ocorrência de falsas memórias em dois tipos diferentes de testes: direto e indireto. Os resultados obtidos estão de acordo com a definição mais popular sobre falsificação de memória (Roediger \& McDermott, 1995), isto é, os participantes recordaram itens que na realidade não foram disponibilizados durante a sessão experimental.

A maioria dos estudos sobre esse assunto concentrou-se em testes diretos, principalmente, no teste de reconhecimento e na recuperação livre (Gallo, Roediger \& McDermott, 2001; Roediger \& McDermott, 1995; Zeelenberg \& cols., 2003). A presente pesquisa avaliou a contribuição do tempo de apresentação como um fator útil na compreensão dos mecanismos cognitivos responsáveis pela produção de falsas memórias. Os resultados atuais replicam o estudo de McDermott e Watson (2001), indo além, pois demonstrou-se que falsas memórias ocorrem também em testes indiretos de identificação perceptual e que esse fenômeno não se restringe apenas a testes que exijam lembrança deliberada. Ou seja, a falsificação de memória pode ocorrer numa amplitude maior da cognição humana. Os dados atuais confirmam os resultados das principais pesquisas da área (McDermott \& Watson, 2001; Roediger \& McDermott, 1995; Seamon \& cols., 1998) de que processos conscientes desempenham um papel central na ocorrência dessas "falhas" mnemônicas; entretanto, essas 
falhas não estão associadas exclusivamente ao processamento consciente como foi evidenciado na presente pesquisa. Este estudo sustenta que a ativação de processos conscientes favorece a produção de falsas memórias mais do que ativações inconscientes. Ou seja, a nossa "certeza" consciente está mais sujeita a "falhas" do que a nossa "intuição". Em outras palavras, é provável que o fenômeno das falsas memórias ocorra em maior escala quando os participantes são instruídos a recordarem de certos itens estudados; por outro lado, quando a instrução não solicita essa evocação deliberada, a produção de falsas memórias diminui consideravelmente.

Os resultados do Experimento 2 confirmaram a hipótese levantada de que a ocorrência de priming da categoria de palavras que indica a produção de falsas memórias, isto é, a média das palavras críticas foi significativamente maior do que as palavras não estudadas, corroborando, assim, também resultados de outros estudos que relataram a ocorrência de priming nas falsas memórias (Marsh, McDermott \& Roediger, 2004; McDermott, 1997). Importante destacar que o resultado obtido neste estudo vai ao encontro aos dados de McDermott (1997), corroborando a hipótese de que a palavra crítica não apresentada é brevemente ativada na fase de estudo, ocorrendo, assim, falsas memórias nos testes indiretos. A teoria da Ativação-Monitoramento (Roediger \& McDermott, 2000) pode explicar os dados obtidos nesse teste de memória implícita. Essa teoria propõe que os mecanismos responsáveis pelas falsas memórias incluem tanto a ativação espalhada quanto o monitoramento. Esses poderiam ocorrer tanto na fase de codificação quanto na fase de evocação. Segundo as evidências apresentadas por vários estudos $(\mathrm{McD}$ ermott \& Watson, 2001; Roediger \& McDermott, 2000), a ativação seria a principal responsável pela etapa de codificação e o monitoramento pelas fases de evocação e codificação. Esses processos não podem ser considerados isoladamente quando se tenta compreender ou explicar a natureza do fenômeno em questão. No caso da identificação perceptual, a palavra crítica não apresentada é ativada diversas vezes, mas tempos breves não permitem que essa ativação chegue ao limiar da consciência. Com o aumento do tempo de apresentação, é provável que processos de monitoramento estratégicos e metacognitivos comecem "discretamente" a supervisionar a identificação das palavras. Isso é evidenciado pelo aumento das médias das palavras críticas não apresentadas nos tempos mais longos em comparação com os tempos breves. Os resultados demonstraram que, variando o tempo de codificação, varia-se de uma forma significativa a produção de falsas memórias. A principal conclusão gerada a partir dessa manipulação é que, quando o participante não tem tempo suficiente para codificar o que estudou, a média de acertos tanto das palavras críticas quanto das palavras estudadas é baixa, sugerindo que o sistema cognitivo não processou adequadamente as informações, evitando também, com isso, as falsificações mnemônicas. A evocação é também um fator importante a considerar, pois há diferenças na produção de falsas memórias quando se instrui um indivíduo a lembrar deliberadamente uma lista de palavras ou quando essa condição não é apresentada. Ou seja, a forma de instrução dirige o processamento de diferentes tipos de estratégias mentais na fase da evocação. No teste de reconhecimento, quando o indivíduo foi solicitado a lembrar as palavras estudadas, houve, possivelmente, um maior envolvimento de processos de monitoramento. Com o aumento do tempo de estudo, esses mecanismos de monitoramento puderam supervisionar melhor o processamento cognitivo e evitar falhas na tomada de decisão ou em algum tipo de mecanismo metacognitivo. Por fim, acredita-se que a teoria de Ativação e Monitoramento é apropriada para tentar entender os mecanismos que produzem falsas memórias em testes diretos e indiretos, porque ela considera, simultaneamente, os processos de codificação e evocação, afirmando uma interdependência dual na produção de falsas memórias.

\section{Considerações Finais}

Por fim, acredita-se que os resultados da presente pesquisa possam contribuir em diversas áreas como, por exemplo, na área clínica, educacional e jurídica. Ilustrando, o nosso sistema jurídico é dependente e aceita relatos de testemunhas oculares e há casos em que o réu é condenado baseando-se no relato de uma única testemunha. Considere a situação na qual uma testemunha identifica o carro de um suspeito na cena do crime, afirmando ter certeza consciente de que viu o carro durante o episódio. Por sua vez, uma segunda testemunha identifica um outro carro de uma forma mais "intuitiva", ou seja, afirmando que o carro em questão não lhe é estranho, mas não consegue ter uma recordação consciente da relação dele com o crime. É possível, nessas circunstâncias, que tenhamos uma inclinação a aceitar o relato da primeira testemunha, porque ela afirmou ter certeza consciente do episódio; entretanto, considerando os resultados deste estudo sobre os efeitos dos tipos de teste e tempo de exposição ao episódio, podemos suspeitar que as "certezas" conscientes da testemunha podem ser, apenas, "certezas" de muitas repetições de ativações na nossa rede semântica. Essa possibilidade questiona o quanto se pode confiar no relato de uma testemunha ocular. Talvez o nosso sistema de crenças favoreça demasiadamente julgamentos racionais, buscando explicações onde não existem (e.g., falácias cognitivas). Pode-se sugerir uma maior atenção ao outro lado da mente humana: sobre o desenvolvimento e compreensão dos nossos julgamentos intuitivos.

\section{Referências}

Arndt, J. \& Hirshman, E. (1998). True and false recognition in MINERVA2: Explanations from a global matching perspective. Journal of Memory and Language, 39(3), 371-391.

Deese, J. (1959). On the prediction of occurrence of particular verbal intrusions in immediate recall. Journal of Experimental Psychology, 58(1), 17-22.

Gallo, D. A., Roediger, H. L. \& McDermott, K. B. (2001). Associative false recognition occurs without liberal criterion shifts. Psychonomic Bulletin \& Review, 8, 579-586.

Gardiner, J. M. (1988). Generation and priming effects in wordfragment completion. Journal of Experimental Psychology: Learning, Memory and Cognition, 14(3), 495-501.

Graf, P. \& Schacter, D. L. (1985). Implicit and explicit memory for new associations in normal and amnesic subjects. Journal of Experimental Psychology: Learning, Memory and Cognition, 11(3), 501-518. 
Hall, J. F. \& Kozloff, E. E. (1973). False recognitions of associates of converging versus repeated words. American Journal of Psychology, 86(1), 133-139.

Hintzman, D. L. (1988). Judgments of frequency and recognition memory in multiple-trace memory model. Psychological Review, 95(4), 528-551.

Hunt, R. R. \& Toth, J. P. (1990). Perceptual identification, fragment completion and free recall: Concepts and data. Journal of Experimental Psychology: Learning, Memory and Cognition, 16(2), 282-290.

Jacoby, L. L. (1983). Remembering the data: Analyzing interactive processes in reading. Journal of Verbal Learning and Verbal Behavior, 22(5), 485-508.

Jacoby, L. L. \& Hollingshead, A. (1989). Direct versus indirect tests of memory for source: Judgments of modality. Journal of Experimental Psychology: Learning, Memory and Cognition, 15(6), 1101-1108.

Jones, T. C. \& Roediger, H. L. III. (1995). The experiential basis of serial position effects. European Journal of Cognitive Psychology, 7(1), 65-80.

Marsh, E. J., McDermott, K. B. \& Roediger, H. L.III (2004). Does test-induced priming play a role in the creation of false memories? Memory, 12(1), 44-55.

McDermott, K. B. (1996). The persistence of false memories in list recall. Journal of Memory and Language, 35(2), 212-230.

McDermott, K. B. (1997). Priming on perceptual implicit memory tests can be achieved through presentation of associates. Psychonomic Bulletin \& Review, 4(4), 582-586.

McDermott, K. B. \& Watson, J. M. (2001). The rise and fall of false recall: The impact of presentation duration. Journal of Memory and Language, 45(1), 160-176.

Nairne, J. S. (1988). The mnemonic value of perceptual identification. Journal of Experimental Psychology: Learning, Memory and Cognition, 14(2), 248-255.

Payne, D. G., Elie, C. J., Blackwell, J. M. \& Neuschatz, J. S. (1996). Memory illusions: Recalling, recognizing and recollecting events that never occurred. Journal of Memory and Language, 35(2), 261-285.

Pinheiro, A. M. V. (1996). Contagem de freqüência de ocorrência de palavras expostas a crianças na faixa pré-escolar e séries iniciais do primeiro grau. São Paulo: Associação Brasileira de Dislexia.

Rajaram, S. (1993). Remembering and knowing: Two means of access to the personal past. Memory and Cognition, 21(1), 89-102.
Ratcliff, R., McKoon, G. \& Verwoerd, M. (1989). A bias interpretation of facilitation in perceptual identification. Journal of Experimental Psychology: Learning, Memory and Cognition, 15(3), 378-387.

Roediger, H. L. (1996). Memory illusions. Journal of Memory and Language, 35(2), 76-100.

Roediger, H. L. \& McDermott, K. B. (1995). Creating false memories: Remembering words not presented in lists. Journal of Experimental Psychology: Learning, Memory and Cognition, 21(4), 803-814.

Roediger, H. L. \& McDermott, K. B. (2000). Tricks of memory. Current Directions in Psychological Science, 9(4), 123-127.

Schacter, D. L., Verfaellie, M. \& Pradere, D. (1996). The neuropsychology of memory illusions: False recall and recognition amnesic patients. Journal of Memory and Language, 35(2), 319-334.

Schiffrin, R. M., Huber, D. E. \& Maritelli, K. (1995). Effects of category length and strength on familiarity in recognition. Journal of Experimental Psychology: Learning, Memory and Cognition, 21(2), 267-287.

Seamon, J. G., Luo, C. R. \& Gallo, D. A. (1998). Creating false memories of words with or without recognition of list items. Psychological Science, 9(1), 20-26.

Stadler, M. A., Roediger, H. L. \& McDermott, K. B. (1999). Norms for word lists that create false memories. Memory and Cognition, 27(3), 494-500.

Stein, L. M. \& Pergher, G. K. (2001). Criando falsas memórias em adultos por meio de palavras associadas. Psicologia: Reflexão e Crítica, 14(2), 353-366.

Tulving, E. (1985). Memory and consciousness. Canadian Psychologist, 26, 1-12.

Underwood, B. J. (1965). False recognition produced by implicit verbal responses. Journal of Experimental Psychology, 70(1), 122-129.

Zeelenberg, R., Plomp, G. \& Raaijmakers, J. G. W. (2003). Can false memories be created through nonconscious processes? Consciousness and Cognition, 12(3), 403-412.

Recebido em 16.11.2006

Primeira decisão editorial em 11.03.2008

Versão final em 20.05.2008

Aceito em 19.06.2008 